\section{Cotrimoxazole-Induced Tremor}

Cotrimoxazole (sulfamethoxazole-trimethoprim) is an antiinfective agent infrequently associated with neurotoxicity. ${ }^{1}$ However, the potential for this medication to cause new symptoms of central nervous system irritability or alteration should not be overlooked, as illustrated by the following case.

A middle-aged patient, weighing $67 \mathrm{~kg}$, was admitted to the intensive care unit (ICU) for respiratory failure.* Cotrimoxazole (1200 mg sulfamethoxazole and $240 \mathrm{mg}$ trimethoprim) IV every $12 \mathrm{~h}$ was initiated for treatment of potential Pneumocystis jirovecii pneumonia. The patient had received a deceased-donor renal transplant 12 years before the current admission to overcome end-stage renal impairment due to immunoglobulin A nephropathy. The patient had been receiving tacrolimus monotherapy as immunosuppression therapy up to the time of admission. The patient had also been receiving oral cotrimoxazole $(800 \mathrm{mg}$ sulfamethoxazole and $160 \mathrm{mg}$ trimethoprim) 3 times a week for prophylaxis before the admission, with concurrent renal function consistent with an estimated glomerular filtration rate of $57 \mathrm{~mL} / \mathrm{min}$.

In the ICU, the patient received 2 IV doses of cotrimoxazole before the medication was switched to oral cotrimoxazole (1600 $\mathrm{mg}$ sulfamethoxazole and $320 \mathrm{mg}$ trimethoprim) twice daily. This approximates doses of $48 \mathrm{mg} / \mathrm{kg}$ daily sulfamethoxazole and $9.6 \mathrm{mg} / \mathrm{kg}$ daily trimethoprim, representing doses lower than usually prescribed for treatment of $P$. jirovecii pneumonia because of an acute decline in renal function (estimated glomerular filtration rate $19 \mathrm{~mL} / \mathrm{min}$ at the time of admission).

Twenty-four hours after receiving the first IV dose, the patient was described as having new-onset twitching of both arms and both legs. By $48 \mathrm{~h}$ of therapy, after the switch to an oral formulation, the twitching had progressed to shaking of the limbs, predominantly the arms. By the fourth day of therapy, the symptoms had progressed to full body tremor. The possible contribution of cotrimoxazole was considered, and the drug was

\footnotetext{
*The patient's consent for publication of this report could not be obtained because of subsequent death from other causes. Personal details not pertinent to understanding of the case have been omitted to protect confidentiality.
}

stopped at this point. Tremors of both the upper and the lower extremities subsequently declined, although complete resolution did not occur until $48 \mathrm{~h}$ after the last dose of cotrimoxazole.

No concurrent drug therapy, electrolyte imbalance, new therapy, or nutritional deficiency could be identified that would have accounted for the onset and offset of the tremor. Concurrent drug therapy included amlodipine, apixaban, acetylsalicylic acid, bisoprolol, hydralazine, insulin, mirtazepine, nitroglycerin, prednisone, and tacrolimus. None of these medications had been initiated recently. Tacrolimus concentrations measured before and subsequent to the symptoms of tremor were 3.7 and $3.3 \mu \mathrm{g} / \mathrm{L}$, respectively (pre-dose measurements), which suggests that tacrolimus was unlikely to have contributed to the symptoms. No computed tomography of the head or electroencephalopathy studies were conducted. The patient was not rechallenged with cotrimoxazole, because other pathogens were isolated from sputum samples, rendering cotrimoxazole therapy unnecessary. The Naranjo score for a potential drug adverse effect was 6 (consisting of 1 point for a previous report of the same effect [as described below], 2 points for symptoms appearing after initiation of the drug, 1 point for improvement with discontinuation, and 2 points for no alternative cause), which suggested a probable cause-effect relationship. No alternative cause, such as drug withdrawal, structural neurological change, or metabolic toxicity, could be found.

Cotrimoxazole has been reported to cause tremor in a wide range of patient populations, from young to elderly patients, ${ }^{2-11}$ and from immunocompetent ${ }^{2}$ to immunosuppressed. ${ }^{3-10}$ Reported associated daily doses have typically been sulfamethoxazole $75-100 \mathrm{mg} / \mathrm{kg}$ daily and trimethoprim $15-20 \mathrm{mg} / \mathrm{kg}$ daily, commonly initiated for treatment of $P$. jirovecii pneumonia $^{3-11}$ or other infections with pathogenic gram-negative bacteria. ${ }^{2}$ No reports are available describing tremor associated with lower doses, as reported in this case. The observed toxicity may be concentration-dependent, given previously described resolution of symptoms with dose reduction. . $8,10^{\circ}$

Onset of the tremor occurs soon after drug initiation; lag periods are reportedly as short as 2 days ${ }^{2,8,11}$ or as long as 8 days, ${ }^{4}$ with 3-5 days being the most common. ${ }^{3-7,9,10}$ Symptoms resolve within 2-3 days following drug discontinuation, ${ }^{3,5-11}$ although 
full resolution has been infrequently reported as taking up to 8-10 days. ${ }^{2,4}$ No additional treatment, beyond simple discontinuation of cotrimoxazole, has been reported as being utilized to quicken the recovery.

The mechanism by which cotrimoxazole causes neurotoxicity is unknown, and which moiety of the combination product causes the toxicity cannot be delineated. Potential mechanisms include accumulation of toxic metabolites due to a glutathione deficiency $^{5}$; inhibition of the enzyme dihydrofolate reductase by trimethoprim, resulting in decreased concentrations of tetrahydrobiopterin, a naturally occurring pteridine that is a cofactor required for the production of catecholamines and serotonin ${ }^{11}$; or inhibition of phenylalanine metabolism resulting in toxic concentrations. ${ }^{8}$

Clinicians should consider the presentation of new-onset tremors, or other neurologic manifestations, as a potential indicator of drug toxicity if cotrimoxazole is being used at the doses considered necessary for treatment of $P$. jirovecii, Stenotrophomonas maltophilia, or Nocardia pneumonia, or if there is significant impairment of renal function. Discontinuation of the drug should result in quick resolution of symptoms without the need for additional corrective therapy. Clinicians should also consider alternative pathogen-directed antimicrobial therapy, because rechallenge of cotrimoxazole at an equivalent dosage has been reported to reproduce the symptoms of tremor. ${ }^{4}$

\section{References}

1. Grill MF, Maganti RK. Neurotoxic effects associated with antibiotic use: management considerations. Br J Clin Pharmacol. 2011;72(3):381-93.

2. Patterson RB, Couchenour RL. Trimethoprim-sulfamethoxazole-induced tremor in an immunocompetent patient. Pharmacotherapy. 1999;19(12): 1456-8.
3. Borucki MJ, Matzke DS, Pollard RB. Tremor induced by trimethoprimsulfamethoxazole in patients with the acquired immunodeficiency syndrome (AIDS). Ann Intern Med. 1988;109(1):77-8.

4. Aboulafia DM. Tremors associated with trimethoprim-sulfamethoxazole therapy in a patient with AIDS: case report and review. Clin Infect Dis. 1996;22(3):598-600.

5. Van Gerpen JA. Tremor caused by trimethoprim-sulfamethoxazole in a patient with AIDS. Neurology. 1997;48(2):537-8.

6. Slavik RS, Rybak MJ, Lerner SA. Trimethoprim/sulfamethoxazole-induced tremor in a patient with AIDS. Ann Pharmacother. 1998;32(2):189-92.

7. Flois-Moore MA, Amodio-Groton MI, Catalano MT. Adverse reactions to trimethoprim/sulfamethoxazole in AIDS. Ann Pharmacother. 2003;37(12): 1810-3.

8. Jundt F, Lempert T, Dörken B, Pezzutto A. Trimethoprim-sulfamethoxazole exacerbates posthypoxic action myoclonus in a patient with suspicion of Pneumocystis jiroveci infection. Infection. 2004;32(3):176-8.

9. Bua J, Marchetti F, Barbi E, Sarti A, Ventura A. Tremors and chorea induced by a trimethoprim-sulfamethoxazole in a child with Pneumocystis pneumonia. Pediatr Infect Dis J. 2005;24(10):934-5.

10. Gray DA, Foo D. Reversible myoclonus, asterixis, and tremor associated with high dose trimethoprim-sulfamethoxazole: a case report. J Spinal Cord Med. 2016;39(1):115-7.

11. Woody RC, Brewster MA. Adverse Effects of trimethoprim-sulfamethoxazole in a child with dihydropteridine reductase deficiency. Dev Med Child Neurol. 1990;32(7):639-42.

Glen Brown, PharmD, FCSHP, BCPS(AQ), BCCCP

Pharmacy

St Paul's Hospital

Vancouver, British Columbia

Glen Brown is also an Associate Editor with the Canadian Journal of Hospital Pharmacy.

Competing interests: None declared.

Funding: None received. 